\title{
Molecular Biomarkers in the Context of Focal Therapy for Prostate Cancer: Recommendations of a Delphi Consensus from the Focal Therapy Society
}

\section{Authors}

Giancarlo Marra ${ }^{1,2}$, Maria Pilar Laguna ${ }^{7}$, Jochen Walz ${ }^{3}$, Christian P. Pavlovich ${ }^{12}$, Fernando Bianco ${ }^{36}$, Justin Gregg ${ }^{41}$, Amir H. Lebastchi ${ }^{37}$, Herbert Lepor ${ }^{38}$, Petr Macek ${ }^{1}$, Soroush Rais-Bahrami ${ }^{9}$, Cary Robertson ${ }^{15}$, Daniel Rukstalis ${ }^{39}$, Georg Salomon ${ }^{40}$, Osamu Ukimura ${ }^{25}$, Andre Luis Abreu ${ }^{22}$, Yann Barbe $^{1}$, Xavier Cathelineau ${ }^{1}$, Giorgio Gandaglia ${ }^{13}$, Arvin K. George ${ }^{11}$, Juan Gomez Rivas ${ }^{5}$, Rajan T.Gupta ${ }^{18^{*}}$, Nathan Lawrentschuk ${ }^{21}$, Veeru Kasivisvanathan ${ }^{29}$, Derek Lomas ${ }^{27}$, Bernard Malavaud ${ }^{8}$, Daniel Margolis ${ }^{31^{*}}$, Yoh Matsuoka ${ }^{19}$, Sherif Mehralivand ${ }^{28}$, Marco Moschini ${ }^{13}{ }^{14}$, Marco Oderda ${ }^{2}$, Hazem Orabi ${ }^{15}$, Ardeshir R. Rastinehad ${ }^{17}$, Mesut Remzi ${ }^{24}$, Ariel Schulman ${ }^{6}$, Toshitaka Shin ${ }^{34}$, Takumi Shiraishi ${ }^{25}$, Abhinav Sidana ${ }^{32}$, Sunao Shoji ${ }^{20}$, Armando Stabile ${ }^{13}$, Massimo Valerio ${ }^{23}$, Varaha S. Tammisetti ${ }^{30}$, Wei Phin Tan ${ }^{15}$, Willemien Van Den Bos ${ }^{35}$, Arnaud Villers ${ }^{16}$, Peter-Paul Willemse ${ }^{26}$, Jean de la Rosette ${ }^{7}$, Thomas Polascik ${ }^{15}$ and Rafael Sanchez-Salas ${ }^{1}$ on behalf of the Focal Therapy Society

\section{Institutions:}

1=Department of Urology, Institut Mutualiste Montsouris, Paris, France

2= Department of Urology, Città della Salute e della Scienza, University of Turin, Turin, Italy

3= Department of Urology, Institut Paoli-Calmettes, Marseille, France

5=Department of Urology, La Paz University Hospital, Madrid, Spain

6=Department of Urology, Maimonides Medical Center, Brooklyn, NY, USA

7=Department of Urology, Medipol Mega University Hospital, Istanbul, Turkey

8=Department of Urology, Institut Universitaire du Cancer Toulouse Oncopole, Toulouse, France

9=Department of Urology, University of Alabama at Birmingham, Birmingham, AL, USA

10=Department of Urology, Oita University Faculty of Medicine, Yufu-shi

Oita, Japan

11= Department of Urology, Division of Urologic Oncology, Michigan Medicine, MI, USA

12= Johns Hopkins University School of Medicine, Baltimore, MD, USA

13= Department of Urology, San Raffaele Hospital, Milan, Italy 
14= Department of Urology, Lucerne Kanton Hospital, Lucerne, Switzerland

15=Department of Urology, Duke University, Durham, NC, USA

16= Department of Urology, CHU de Lille, Lille, France

17= Department of Urology, Lenox Hill Urology, NY, USA

18= Department of Radiology, Duke University, Durham, NC, USA

19= Urology at Tokyo Medical and Dental University, Tokyo, Japan

20= Department of Urology, Tokai University Hachioji Hospital, Hachioji, Tokyo, Japan

21= Department of Urology, Peter MacCallum Cancer Center, Melbourne, VIC, Australia

22= Department of Urology, Keck School of Medicine and University of South California, CA, USA

23= Department of Urology, Centre Hospitalier Universitaire Vaudois, Lausanne, Switzerland

24= Department of Urology, Döbling Hospital, Vienna, Austria

25= Department of Urology, Kyoto Prefectural University of Medicine, Kyoto, Japan

26=Department of Urology, Erasmus Medical Center, Rotterdam, The Netherlands

27= Department of Urology, Mayo Clinic, Rochester, MN, USA

28= Urologic Oncology Branch, National Cancer Institute, National Institutes of Health, Bethesda, Maryland

29= Department of Urology, University College London Hospitals, London, UK

30= Department of Radiology and Radiological Sciences, Vanderbilt University Medical Center, Nashville, TN, USA

31= Department of Radiology, Weill Cornell Imaging, Cornell University, New York, NY, USA

32= Division of Urology, University of Cincinnati College of Medicine, Cincinnati, OH, USA

34= Department of Urology, Oita University, Oita, Japan

35= Academic Medical Centre, University of Amsterdam, Amsterdam, The Netherlands

36=Urological Research Network, Nova University, Miami, FL, USA

37= Department of Urology, University of Michigan, Ann Arbor, Michigan, USA 
38= Department of Urology, NYU Langone Medical Center, New York, NY

39= Department of Urology, Wake Forest Baptist Medical Center, Winston-Salem, North Carolina, USA

40= Martini Clinic, Prostate Cancer Center, Hamburg, Germany

41= Department of Urologic Surgery, Vanderbilt University Medical Center, Nashville, Tennessee, USA

\begin{abstract}
Background: Focal Therapy (FT) for Prostate Cancer (PCa) is promising. However, long-term oncological results are awaited and there is no consensus on follow-up strategies. Molecular biomarkers $(\mathrm{MB})$ may be useful in selecting, treating and following up men undergoing $\mathrm{FT}$, though there is limited evidence in this field to guide practice. We aimed to conduct a consensus meeting, endorsed by the Focal Therapy Society, amongst a large group of experts, to understand the potential utility of MB in FT for localised PCa. Materials and Methods: A 38-item questionnaire was built following a literature search. The authors then performed three rounds of a Delphi Consensus using DelphiManager, using the GRADE grid scoring system, followed by a face-to-face expert meeting. Three areas of interest were identified and covered concerning $M B$ for $F T$, i) the current/present role; ii) the potential/future role; iii) the recommended features for future studies. Consensus was defined using a 70\% agreement threshold. Results: Of 95 invited experts, 42 (44.2\%) completed the three Delphi rounds. Twenty-four items reached a consensus and they were then approved at the meeting involving $(n=15)$ experts. Fourteen items reached a consensus on uncertainty, or they did not reach a consensus. They were re-discussed, resulting in a consensus $(n=3)$, a consensus on a partial agreement $(n=1)$, and a consensus on uncertainty $(n=10)$. A final list of statements were derived from the approved and discussed items, with the addition of three generated statements, to provide guidance regarding MB in the context of FT for localised PCa. Research efforts in this field should be considered a priority. Conclusion: The present study detailed an initial consensus on the use of MB in FT for PCa. This is until evidence becomes available on the subject.
\end{abstract}

Keywords: prostate cancer, localised, focal therapy, molecular biomarker, Delphi 


\section{Corresponding Author:}

Doctor Rafael Sanchez-Salas

Department of Urology

Institut Mutualiste Montsouris

75014 Paris,

France

Email: Rafael.sanchez-salas@imm.fr

\section{Manuscript}

\section{Introduction}

Focal therapy (FT) for prostate cancer (PCa) is an emerging treatment option, introduced to reduce the side effects of radical treatments, whilst maintaining a similar oncological control ${ }^{1,2}$.

Currently, guidelines suggest FT to be restricted to clinical trials and prospective registries ${ }^{3}$. Absence of long-term results and of randomized controlled trials (RCT) against radical treatments remain amongst the major arguments hampering its introduction in clinical practice ${ }^{2,4}$.

On the one hand, complications are low and short-to-medium term results are favourable ${ }^{2,5}$. On the other hand, up to one in five men has a disease recurrence or persistence if prostate biopsies are performed at 12 months after treatment ${ }^{3}$, and approximately $50 \%$ may need at least one retreatment within five years ${ }^{6}$. Re-do treatments may have low morbidity and salvage radical prostatectomy following FT, if performed in expert hands, may be comparable to a first-line setting except for the poor post-operative erectile function ${ }^{7}$. A critical assessment of FT outcomes clearly shows that it is still far from achieving optimal oncological control, and the urological community should strive to identify and implement the weak points seeking for improvement ${ }^{8,9}$.

Amongst the possible reasons for disease recurrence/persistence after FT are: i) an inappropriate ablation leaving PCa untreated due to technical and/or operator-dependent issues; ii) incorrect targeting either due to limitations of the imaging modality to identify the precise boundaries of each tumor or the inability of the chosen imaging modality to identify the tumor itself (eg MRI-invisible tumor); iii) the PCa microenvironment being modified in a pro-tumorigenic fashion by treatmentinduced inflammation and possibly favouring the development of resistant clinically significant PCa clones; and iv) inappropriate patient selection due to the limitations of the current risk stratification tools ${ }^{8}$.

The latter, in particular, may lead to undertreatment of high-risk disease, significantly increasing the risk of recurrence/progression after FT when compared to intermediate-risk $\mathrm{PCa}^{6,10}$, to underestimation of the disease volume, and to underdetection of clinically significant disease in the non-ablated prostate. 
Molecular Biomarkers (MB) have been introduced in clinical practice, in order to provide a more precise, patient-based risk stratification. Several validated kits are commercially available for PCa diagnostic, prognostic, and in adjuvant treatment settings. These include blood, urine, and prostate tissue based-markers, which evaluate a wide variety of cellular pathways involved in the prostate cancer pathway, from baseline gene mutations to RNA, methylation patterns, and protein expression levels ${ }^{11-13} . \mathrm{MB}$ have been mainly studied in the context of active surveillance, with the aim of identifying patients who would benefit the most from treatment. They have also been studied in a first-line diagnostic or post-prostatectomy context, to identify those at high risk of developing PCa metastases, and eventually, to identify those who might benefit from adjuvant treatment $^{11-13}$.

Nonetheless, little if no evidence exists on the use of MB in the context of FT, whether to improve risk stratification, to reduce an inappropriate treatment delivery, or to improve outcomes. Furthermore, the lack of criteria/biomarkers to reliably follow-up patients receiving FT and even patients simply on active surveillance is well acknowledged.

With this in mind, a Delphi Consensus was devised and conducted by the Focal Therapy Society (FTS) aiming to frame the current and future potential role of MB in the context of FT for PCa and to ascertain needs of clinicians regarding MB application in the FT context going forward. We hereby aim to describe the results and recommendations of this Consensus.

\section{Material and Methods}

In 2019, a Delphi consensus on the role of Biomarkers in FT of PCa was endorsed by the FTS. The 'a priori' study methodology comprised of three phases.

\subsection{Phase I: Literature Review and Questionnaire Design}

A non-systematic review of the English language literature was conducted by two authors (G.M. and R.S.S.) on the $5^{\text {th }}$ January 2020 using the PubMed portal, using the terms "focal therapy" AND "prostate cancer" AND "biomarkers", to summarise the current evidence on MB and their current and potential use in FT. No papers investigating MB in the context of FT for PCa were identified.

Two authors designed a 38-item questionnaire that covered three main areas concerning the current and future use of MB in FT for PCa:

1) Current evidence and the role of $M B$;

2) Future/Potential role of $M B$;

3) Important tests to be included in future studies for assessing the role of MB.

The literature review summary, the preliminary survey design, and the Delphi methodology were evaluated by an advisory panel composed of 2 opinion leaders in FT (M.P.L., T.P.) and by a team of dedicated methodologists (Department of Trials Methodology, Liverpool Clinical Trials Centre, Liverpool University, Liverpool, UK). The panel was asked to review the questionnaire, integrating any other points if necessary, and to implement/comment on the methodology, before starting the consensus rounds. The questions/items in their final form are shown in Supplementary Material 1. The definitions used in the consensus are displayed in Table 1. 


\subsection{Phase II: Delphi Consensus}

All of the included statements were entered into DelphiManager, a bespoke online tool, in order to perform the Delphi Consensus ${ }^{14}$.

The survey was sent to Urologists, Radiologists and Pathologists who are experts in the field of FT. An expert was defined as having performed at least 100 FT cases with one or multiple energies and/or having published at least 10 PCa papers one of whom in the field of FT. As FT is currently focused on localised PCa treatments, no Oncologists were included. Since FT is not recognized as a standard of care, patient representatives were not included.

The levels of agreement were based on a nine-point scale, according to the GRADE grid $^{14,15}: 1-3$ disagree; 4-6 uncertain; and 7-9 agree. An "Unable to Score" option was also added in the case of insufficient knowledge/expertise. Consensus was defined when at least $70 \%$ of the participants were agreeing (score 1-3), being uncertain (score 4-6), or disagreeing (score 7-9) as previously described ${ }^{14,15}$. However, as the field of biomarkers in FT is currently unexplored, no concomitant $\leq 15 \%$ disagreement threshold was used.

Repeated anonymous voting was performed in three rounds ( $1^{\text {st }}$ round: 16 th- $27^{\text {th }}$ January $2020 ; 2^{\text {nd }}$ round: 28 January- $2^{\text {nd }}$ February 2020; $3^{\text {rd }}$ round $3^{\text {rd }}-7^{\text {th }}$ February 2020). After the first round, the questions reaching consensus were removed from the subsequent round and the participants were provided with the results from the previous round, in the form of histograms plus percentages - See example in Supplementary Material 2. Those items that reached a consensus of agreeing or disagreeing with a statement were presented for approval but not re-discussed in Phase III. Conversely, the items reaching a consensus on uncertainty towards a statement and the items not reaching a consensus were re-discussed in Phase III.

\subsection{Phase III: Face-to-face expert meeting}

The last phase included a face-to-face consensus meeting during the Focal Therapy Society Congress, $9^{\text {th }}$ February, 2020, in Washington D.C., USA. The discussion was chaired by two Urologists (R.S.S., G.M.) and included 15 experts.

The voting panel consisted of the 15 participants (Urologists). The statements reaching consensus by the $3^{\text {rd }}$ survey round (agreement or disagreement), were presented for approval and all of the statements not reaching consensus or uncertainty after the three Delphi rounds were formally rediscussed (Figure 1). The anonymous voting was performed live during the meeting through Poll Everywhere Voting Software (https://www.polleverywhere.com/, last accessed, 11th February 2020) by using individual smartphone devices. Each participant was asked to provide a score to approve, disapprove, or express uncertainty, towards a statement/item.

For inclusion in the final recommendations, each survey item was required to have reached group consensus by the end of the three survey rounds (Phase II), plus approval at the face-to-face meeting (Phase III), or consensus at the face-to-face meeting (Phase III). All of the meeting discussions and the statements were recorded.

The resulting document was distributed for approval to all survey participants that completed the 3 Delphi rounds and to face-to-face meeting attendees. 


\section{Results}

The Study Flow Chart and an overview of the Delphi results are provided in Figure 1. From the 95 invited experts, 54 (56.8\%), 46 (48.4\%) and 42 (44.2\%) completed the first, second and third round respectively (third round $n=40$ Urologists; $n=2$ Radiologists). Overall, consensus was reached in 29 of 38 items (76.3\%) across the three rounds (Table 2, 3 and 4). The distribution of the items reaching consensus was: agreement in 19 items, disagreement in 5 and uncertainty in 5 items.

Consensus was reached in 14/38, 9/24 and 6/15 items in the first, second and third round respectively. Overall, the 24 items were scored in agreement or disagreement with consensus at the Delphi-were presented and approved during the face to face meeting and approved. From the 14 items that did not reach consensus or were scored with uncertainty and consensus, 3 were scored in agreement but without consensus and in 1 partial agreement but without consensus (See statement 3.3 - Table 5) was reached. For the remaining 10, there was agreement regarding uncertainty and thus no specific statements could be made. During the face-to-face meeting, 3 new statements were generated and added to the final list of statements. The final list of statements is displayed in Table 5.

\subsection{Current Evidence/Role of MB in the context of FT for localised PCa}

Six of the eight formulated items found agreement in the three Delphi rounds, leading to six statements on the current role/evidence of MB (Table 2). An overall agreement was found on the lack of evidence supporting the present role of $\mathrm{MB}$, their routine use and their impact on clinical decision making. Multi-parametric magnetic resonance imaging ( $\mathrm{mpMRI}$ ) was recognised as more accessible and useful than MB in the current FT scenario (Table 5-1.1 to 1.3.2).

\subsection{Future/Potential Role of MB in the context of FT for localised PCa}

\subsubsection{Single MBs}

Overall, items 9 to 21 included a list of single MB (Table 3). Only three items found consensus; PSA and PSAd were recognised as having a current role (Table 5 - 2.2.1, 2.2.2 -) whilst there was a consensus on the lack of interest towards the application of PCA3 in the context of FT (Table $\mathbf{5}$ 2.3.1). On the contrary, consensus on uncertainty $(n=5)$ or no consensus $(n=5)$ (Table 3 ) was present on the majority of items, as no other available MB have a proven impact in the field of FT. During the meeting, the panel agreed on uncertainty for all of these items; consequently, no statements were made on the single MBs.

\subsubsection{MBs overall}

Three statements were generated and added by the panel (Table 5 - $2.1 ; \mathbf{2} .4 .1 ; \mathbf{2} .4 .2$ ). The panel recognised that research in the field is a priority. Whilst no preferred source (tissue or biological fluids) for $M B$ was identified in a diagnostic setting, there was an agreement in ideally preferring non-tissue based MB in the follow-up setting after FT treatment.

\subsubsection{Ideal Features and Endpoints for MB}


Full agreement was reached during the Delphi rounds and this was confirmed by the panel on the six items that concerned ideal features for MB in the context of FT for localised PCa (Table 4 and Table 5 - 2.5.1 to 2.5.6). The ideal features/endpoints included: i) an accuracy improvement over currently available diagnostic tools; ii) the ability to rule out high risk disease; iii) the ability to identify men with intermediate risk disease; iv) the ability to rule out intermediate risk disease outside of the treated/target area; the ability to predict a high risk of PCa recurrence/persistence v) overall, and vi) requiring a radical treatment; vii) the ability to decrease PCa recurrence/persistence when incorporated in clinical decision-making.

\subsection{Tests/Exams in Future Studies to assess the role of MB in the context of FT for localised PCa}

Five of 10 items concerning the ideal tests/exams that should be included in future studies for assessing the role of $\mathrm{MB}$ in the context of FT reached agreement during the Delphi survey rounds (Table 5 - 3.1.1 to 3.2.5). The panel agreed that digital rectal examinations (Table 5 - 3.1.6) should also be included as a relevant test. On the contrary there was consensus that 3 items, Choline C-11 PET scan, CT scan, and bone scan (Table 5 - 3.2.1 to 3.2.3) should not be used as standard for comparison when assessing the usefulness of a MB in a FT context.

The use of PSMA-PET in the context of MB for PCa FT (Table 5 - 3.3) was found to be of interest by the panel (Table 5 - 3.3).

\section{Discussion}

The present study reports on the first Delphi Consensus evaluating the role of $\mathrm{MB}$ in the context of FT for localised PCa. Currently, no evidence concerning in this field is present. Our work pioneered this area aiming to: i) clarify the current role of $M B$ in routine clinical practice when involving urologists using $\mathrm{FT}$; ii) define the main ideal features for future studies aiming to deal with this subject when involving researchers, funders, and industry.

\subsection{Current role of $\mathrm{MB}$ in the context of FT for PCa}

The first key point was the unanimous acknowledgement that there was no evidence surrounding the use of MB in the context of FT for PCa.

This derived both from the absence of any relevant articles being found during the literature search process and from the personal experience of panel and Delphi members. Despite FT has good oncological results for low- to intermediate risk PCa on the medium term ${ }^{6,10,16,17}$, a room for improvement exists. Optimization of FT patient selection and timely detection of treatment failures/relapses through tools other than those currently avaible is indeed key.

However, MB have been evaluated in different contexts than FT (e.g. active surveillance, radical prostatectomy and following chemotherapy) and have frequently not been adjusted for the current risk stratification/diagnostic pathway. Namely, mpMRI and mpMRI-targeted biopsies were not performed in the majority of the cohorts investigating and/or validating $\mathrm{MB}^{11-13,18}$. Pre-biopsy mpMRI and mpMRI-targeted biopsies in the case of a suspicious imaging result ${ }^{19}$ are now recommended by the major guidelines and they constitute "de facto" the basis -of FT feasibility for PCa. Hence, for all of these reasons, until supporting evidence becomes available, a patient should not be offered, or precluded, from gland preserving strategies that are based on MB. 


\subsection{Future/potential role of MB in the context of FT for PCa}

The second important finding was that tools to correctly allocate (diagnostic setting - before FT) and safely monitor (follow-up setting - after FT) FT patients are urgently awaited beyond mpMRI. Consequently research in the field of MB FT related should be considered a priority. The outcomes of the consensus underscore the need for collaboration among the different stakeholders involved in the field, and the implementation of "smart" research protocols while partnering with the industry. FT has been proven safe, with improved functional outcomes compared. Nonetheless, oncological outcomes are suboptimal, and improving patient selection is perfunctory to decrease the recurrence rates ${ }^{6,10,16}$. In addition, especially when in-field, the disease relapse/persistence may harbour aggressive cancer. Thus, early recognition may translate into therapeutic benefit ${ }^{7,20-22}$

The third point was the uncertainty on-which biomarkers will likely have a role in the context of FT. Neither agreement nor disagreement was reached for the majority of the currently commercially available MB, either during the Delphi rounds or in the face-to face meeting. In spite of a lively discussion on each single available test, we could not formulate any statement on which test/s may be worth investigating in future studies. However, overall, the panel agreed there was uncertainty and this remains to be addressed.

It is worth noting that two statements on the ideal MB source were added to the initial Consensus items. As PCa diagnosis relies on histology, with Gleason score being fundamental in risk stratification, there was no preference for tissue, blood, urine, or other marker sources in the diagnostic setting. On the contrary, in the follow-up setting, a non-invasive MB (biological fluids easy to obtain) that allows for reducing or avoiding monitoring biopsies, would be preferred over a tissuebased molecular test. When considering that PSA changes can be challenging to interpret when evaluating for recurrence of $\mathrm{PCa}$ after $\mathrm{FT}$, and that mpMRI warrants further investigation in the follow-up setting, the search for an adequate biomarker capable to monitor progession after FT becomes an important issue.

A fourth relevant point of discussion was the definition of the endpoints that will need to be addressed by future research on $\mathrm{MB}$ in the context of FT. Intermediate risk disease remains the current ideal FT candidate but it has a relatively slow evolving course, even when left untreated ${ }^{23}$. Thus, intermediate surrogate endpoints would likely allow for short-to-medium term answers on a potential role for biomarkers. In particular, a key achievement will be to impact on clinical decisionmaking, allowing for a reduction in the number of in- and out-of field persistence/recurrence, while providing timely information on persistences/recurrences post-treatment, reducing or virtually avoiding, the control biopsies.

Lastly, a MB will need to demonstrate its independent ability to improve the current pathway. A fairly strict list on what will be the standards for comparison to determine the prognostic or predictive ability of the chosen $\mathrm{MB}$ regarding risk-groups categorization and response to treatment has been devised for oncoming studies. Interestingly, while PSMA-PET was not considered amongst the recommended tests for comparison as still subject to accessibility issues and pendent of validation ${ }^{24-28}$, it is increasingly used in a treatment-naïve context and was felt of interest.

Multicenter trials are ongoing or about to start in the field of FT or MB ${ }^{29-31}$. Our consensus provides scientific and practical information, obtained from an international expert panel through a validated methodology, to implement $\mathrm{MB}$ in these studies, possibly providing and/or 
planning to obtain timely evidence to further improve our current outcomes.

Some limitations in this study have to be acknowledged. First, the work produced statements based on an expert consensus, which represents the lowest level of evidence. However, with exclusion of the present report, the field remains unexplored. Second, a literature review summary concerning current evidence on MB in the context of FT for PCa did not provide any published studies on this topic. Third, no panelists other than urologists were present at the face-to-face meeting. The three Delphi rounds were completed mainly by urologists and by some radiologists. This limits the generazability of the findings in a multi-stakeholder context. However, it may be partly related-to chance (the non-attending/non-replying invited non-urological experts) and because the target audience of the first FTS meeting being mainly urological. Similarly, no patient representatives were included, mainly due to the developmental nature of FT. Finally, we could not find clear positions and/or agreement on some items. This uncertainty, which is linked to the absence of evidence, will hopefully be addressed by future research on MB in the context of FT.

\section{Conclusions}

At present, there is limited evidence regarding the use of MB in the context of FT for PCa. A Delphi Consensus, including a group of experts in the field, was therefore performed in order to produce consensus recommendations on this subject. Currently, no evidence supports the use of MB in the field of FT. Thus, MB should not be routinely offered in this field. Nonetheless, tools to correctly allocate (before FT) and safely monitor (after FT) FT patients are urgently awaited. MB should play an important role in future research in FT and should be considered a priority. Meaningful endpoints should be chosen to identify a molecular biomarker that will objectively improve the current FT pathway in a clinically significant manner compared to to existing options.

Ethics: the present study did not involve patients. No ethical committee approval was needed.

Conflict of Interests: none to declare.

Funding: the present study has been endorsed and funded by the Focal Therapy Society. Giancarlo Marra's research work at Institut Mutualiste Montsouris has been supported by a scholarship from the European Urological Scholarship Programme (EUSP).

Authors contributions: All authors significantly contributed to the manuscript. Giancarlo Marra and Rafael Sanchez-Salas were involved in study design, data acquisition, statistical analysis and in drafting the manuscript. Giancarlo Marra, Maria Pilar Laguna, Jochen Walz' Christian P. Pavlovich, Fernando Bianco, Justin Gregg, Amir H. Lebastchi, Herbert Lepor, Petr Macek, Soroush RaisBahrami, Cary Robertson, Daniel Rukstalis, Georg Salomon, Osamu Ukimura and Rafael SanchezSalas participated to the face-to-face consensus meeting during the Focal Therapy Society Congress in Washington DC, US. All authors successfully completed all the three rounds of the Delphi consensus and read and approved the last version of the manuscript. 


\section{Figure and Tables Legend}

\section{Figure and Tables Legend}

Figure 1. Study Flow Chart.

Table 1. Definitions used in the Delphi rounds and in the face-to-face consensus meeting.

Table 2. Delphi Items to reach consensus statements on the current role of Molecular Biomarkers in the context of Focal Therapy for Prostate Cancer. Scores in the three Delphi rounds and final outcomes. MB=Molecular Biomarkers; FT=Focal Therapy; PCa=Prostate Cancer; UTS=Unable to Score; NC=not completed; Green= consensus on agreement; Red=consensus on disagreement; Yellow=consensus on uncertaninty.

Table 3. Delphi Items to reach consensus statements on the current role of Molecular Biomarkers in the context of Focal Therapy for Prostate Cancer. Scores in the three Delphi rounds and final outcomes. $\mathrm{MB}=$ Molecular Biomarkers; $\mathrm{FT}=$ Focal Therapy; $\mathrm{PCa}=$ Prostate Cancer; UTS=Unable to Score; NC=not completed; RT=radical treatment. Green= consensus on agreement; Red=consensus on disagreement; Yellow=consensus on uncertaninty.

Table 4. Delphi Items to reach consensus statements on important tests that have to be included in future studies evaluating the role of Molecular Biomarkers in the context of focal therapy for localised Prostate Cancer. ${ }^{*}=$ studies assessing the role of Molecular Biomarkers in the context of focal therapy for localised prostate cancer should also include/allow comparison of the Molecular Biomarker'; MB=Molecular Biomarkers; FT=Focal Therapy; PCa =Prostate Cancer; UTS=Unable to Score; $\mathrm{NC}=$ not completed; $\mathrm{DRE}=$ Digital Rectal Examination; Green= consensus on agreement; Red=consensus on disagreement; Yellow=consensus on uncertaninty.

Table 5. Focal Therapy Society Statements on Molecular Biomarkers in the context of Focal Therapy for Prostate Cancer. MB=Molecular Biomarkers; FT=Focal Therapy; $\mathrm{PCa}=$ Prostate Cancer. For the definitions used, please see Table $1 .{ }^{*}=$ statements generated and added by the consensus panel at the face-to-face meeting.

Supplementary Material 1. Delphi questionnaire.

Supplementary Material 2. Example of the graph being shown during the second and third Delphi round to summarise the results from the previous round.

\section{References}

1. Marra G, Ploussard G, Ost P, et al: Focal therapy in localised prostate cancer: Real-world urological perspective explored in a cross-sectional European survey. Urol. Oncol. Semin. Orig. Investig. 2018; 36: 529.e11-529.e22. Available at: https://doi.org/10.1016/j.urolonc.2018.08.013.

2. Marra G, Gontero P and Valerio M: Changing the prostate cancer management pathway: why Focal Therapy is a step forward. Arch. Esp. Urol. 2016; 69: 271-80. Available at: 
http://www.ncbi.nlm.nih.gov/pubmed/27416630, accessed September 8, 2019.

3. van der Poel HG, van den Bergh RCN, Briers E, et al: Focal Therapy in Primary Localised Prostate Cancer: The European Association of Urology Position in 2018. Eur. Urol. 2018; 74: 84-91. Available at: http://www.ncbi.nlm.nih.gov/pubmed/29373215, accessed September 8, 2019.

4. Gontero P, Marra G, Teber D, et al: Making a case "against" focal therapy for intermediaterisk prostate cancer. World J. Urol. 2020. Available at: https://pubmed.ncbi.nIm.nih.gov/32529451/, accessed September 30, 2020.

5. Valerio M, Cerantola Y, Eggener SE, et al: New and Established Technology in Focal Ablation of the Prostate: A Systematic Review. Eur. Urol. 2017; 71: 17-34. Available at: http://www.ncbi.nlm.nih.gov/pubmed/27595377, accessed September 8, 2019.

6. Stabile A, Orczyk C, Hosking-Jervis F, et al: Medium-term oncological outcomes in a large cohort of men treated with either focal or hemi-ablation using high-intensity focused ultrasonography for primary localized prostate cancer. BJU Int. 2019; 124: 431-440. Available at: http://www.ncbi.nlm.nih.gov/pubmed/30753756, accessed September 8, 2019.

7. Marra G, Gontero P, Walz JC, et al: Complications, oncological and functional outcomes of salvage treatment options following focal therapy for localized prostate cancer: a systematic review and a comprehensive narrative review. World J. Urol. 2019. Available at: https://doi.org/10.1007/s00345-019-02642-9.

8. Tourinho-Barbosa RR, Sanchez-Salas R, Claros OR, et al: Focal Therapy for Localized Prostate Cancer with Either HIFU or Cryoablation: A Single Institution Experience. J. Urol. 2019.

9. Marra G, Dell'oglio P, Baghdadi M, et al: Multimodal treatment in Focal Therapy for localized prostate cancer using concomitant short-term androgen deprivation therapy: the ENHANCE prospective pilot study. Minerva Urol. Nefrol. 2019. Available at: http://www.ncbi.nlm.nih.gov/pubmed/31508924, accessed October 10, 2019.

10. Oishi M, Gill IS, Tafuri A, et al: Hemigland Cryoablation of Localized Low, Intermediate and High Risk Prostate Cancer: Oncologic and Functional Outcomes at 5 Years. J. Urol. 2019; 202: 1188-1198.

11. Cucchiara V, Cooperberg MR, Dall'Era M, et al: Genomic Markers in Prostate Cancer Decision Making. Eur. Urol. 2018; 73: 572-582. Available at: http://dx.doi.org/10.1016/j.eururo.2017.10.036.

12. Moschini M, Spahn M, Mattei A, et al: Incorporation of tissue-based genomic biomarkers into localized prostate cancer clinics. BMC Med. 2016; 14: 1-7. Available at: http://dx.doi.org/10.1186/s12916-016-0613-7.

13. Eggener SE, Rumble RB, Armstrong AJ, et al: Molecular Biomarkers in Localized Prostate Cancer: ASCO Guideline. J. Clin. Oncol. 2019: JCO1902768. Available at: http://www.ncbi.nlm.nih.gov/pubmed/31829902.

14. Horwich A, Babjuk M, Bellmunt J, et al: EAU-ESMO consensus statements on the management of advanced and variant bladder cancer-an international collaborative multistakeholder effort: under the auspices of the EAU and ESMO Guidelines Committeest. Ann. Oncol. Off. J. Eur. Soc. Med. Oncol. 2019; 30: 1697-1727.

15. Lam TBL, MacLennan S, Willemse PPM, et al: EAU-EANM-ESTRO-ESUR-SIOG Prostate Cancer Guideline Panel Consensus Statements for Deferred Treatment with Curative Intent for 
Localised Prostate Cancer from an International Collaborative Study (DETECTIVE Study). Eur. Urol. 2019; 76: 790-813.

16. Tourinho-Barbosa RR, Sanchez-Salas R, Claros OR, et al: Focal Therapy for Localized Prostate Cancer with Either HIFU or Cryoablation: A Single Institution Experience. J. Urol. 2019: 101097JU0000000000000506. Available at: http://www.ncbi.nlm.nih.gov/pubmed/31437121, accessed September 8, 2019.

17. Shah TT, Peters M, Eldred-Evans D, et al: Early-Medium-Term Outcomes of Primary Focal Cryotherapy to Treat Nonmetastatic Clinically Significant Prostate Cancer from a Prospective Multicentre Registry. Eur. Urol. 2019; 76: 98-105. Available at: https://doi.org/10.1016/j.eururo.2018.12.030.

18. Carneiro A, Priante Kayano P, Gomes Barbosa ÁR, et al: Are localized prostate cancer biomarkers useful in the clinical practice? Tumor Biol. 2018; 40: 1-19.

19. Marra G, Ploussard G, Futterer J, et al: Controversies in MR targeted biopsy: alone or combined, cognitive versus software-based fusion, transrectal versus transperineal approach? World J. Urol. 2019; 37: 277-287.

20. Thompson JE, Sridhar AN, Tan WS, et al: Pathological Findings and Magnetic Resonance Imaging Concordance at Salvage Radical Prostatectomy for Local Recurrence following Partial Ablation Using High Intensity Focused Ultrasound. J. Urol. 2019; 201: 1134-1143. Available at: http://www.ncbi.nlm.nih.gov/pubmed/30730409, accessed October 26, 2019.

21. Marconi L, Stonier T, Tourinho-Barbosa R, et al: Robot-assisted Radical Prostatectomy After Focal Therapy: Oncological, Functional Outcomes and Predictors of Recurrence(Figure presented.). Eur. Urol. 2019; 76: 27-30.

22. Palermo $G$, Bassi $P$, Racioppi $M$, et al: Circulating tumor cells as prognostic biological marker in different stages prostate cancer and the effect of different therapeutic approaches on their expression. Minerva Urol. e Nefrol. 2020; 72: 214-222. Available at: https://pubmed.ncbi.nlm.nih.gov/31144490/, accessed September 22, 2020.

23. Neal DE, Metcalfe C, Donovan JL, et al: Ten-year Mortality, Disease Progression , and Treatment-related Side Effects in Men with Localised Prostate Cancer from the ProtecT Randomised Controlled Trial According to Treatment Received. Eur. Urol. 2019: 1-11. Available at: https://doi.org/10.1016/j.eururo.2019.10.030.

24. Corfield J, Perera M, Bolton D, et al: 68Ga-prostate specific membrane antigen (PSMA) positron emission tomography (PET) for primary staging of high-risk prostate cancer: a systematic review. World J. Urol. 2018; 36: 519-527.

25. Han S, Woo S, Kim YJ, et al: Impact of 68Ga-PSMA PET on the Management of Patients with Prostate Cancer: A Systematic Review and Meta-analysis. Eur. Urol. 2018; 74: 179-190.

26. Perera M, Papa N, Roberts M, et al: Gallium-68 Prostate-specific Membrane Antigen Positron Emission Tomography in Advanced Prostate Cancer-Updated Diagnostic Utility, Sensitivity, Specificity, and Distribution of Prostate-specific Membrane Antigen-avid Lesions: A Systematic Review and Meta-analysis. Eur. Urol. 2019.

27. Morigi JJ, Anderson J, De Nunzio C, et al: PSMA PET/CT and staging high risk prostate cancer: a non-systematic review of high clinical impact literature. Minerva Urol. Nefrol. 2020. Available at: https://pubmed.ncbi.nlm.nih.gov/32550630/, accessed September 22, 2020.

28. Heetman JG, Lavalaye J, van Selm S, et al: Is there any additional value to 68Ga-PSMA 
$\mathrm{PET} / \mathrm{CT}$ in patients with suspicion of prostate cancer despite negative MRI and systematic biopsy? Minerva Urol. Nefrol. 2020; 72: 511-513. Available at:

https://pubmed.ncbi.nlm.nih.gov/32284529/, accessed September 22, 2020.

29. Reddy D, Shah TT, Dudderidge T, et al: Comparative Healthcare Research Outcomes of Novel Surgery in prostate cancer (IP4-CHRONOS): A prospective, multi-centre therapeutic phase II parallel Randomised Control Trial. Contemp. Clin. Trials 2020: 105999.

30. Anon: https://clinicaltrials.gov/ct2/show/NCT04063566. Last accessed 15 November, 2020.

31. Anon: https://clinicaltrials.gov/ct2/show/NCT03668652 . Last accessed 15 November 2020. 
Table 1. Definitions used in the Delphi rounds and in the Face to face consensus meeting

- Molecular Biomarker: A biologic molecule found in human tissues and/or fluids able to provide information on the studied disease (diagnostic - presence or absence; prognostic disease aggressiveness; likelihood of response to treatment).

- Focal therapy for Prostate cancer: An ablation/treatment not targeting the whole prostate gland but treating the area containing prostate cancer, plus a safety margin, whilst sparing the remaining benign prostatic tissue.

- High risk prostate cancer: Gleason Score $\geq 8$ and/or cT3a disease and/or PSA $>20 \mathrm{ng} / \mathrm{mL}$.

- Localized prostate cancer: Prostate cancer localized within the prostate gland and with no evidence of systemic spread. 
Table 2- Current Evidence/Role of Molecular Biomarkers in the context of FT for Pca

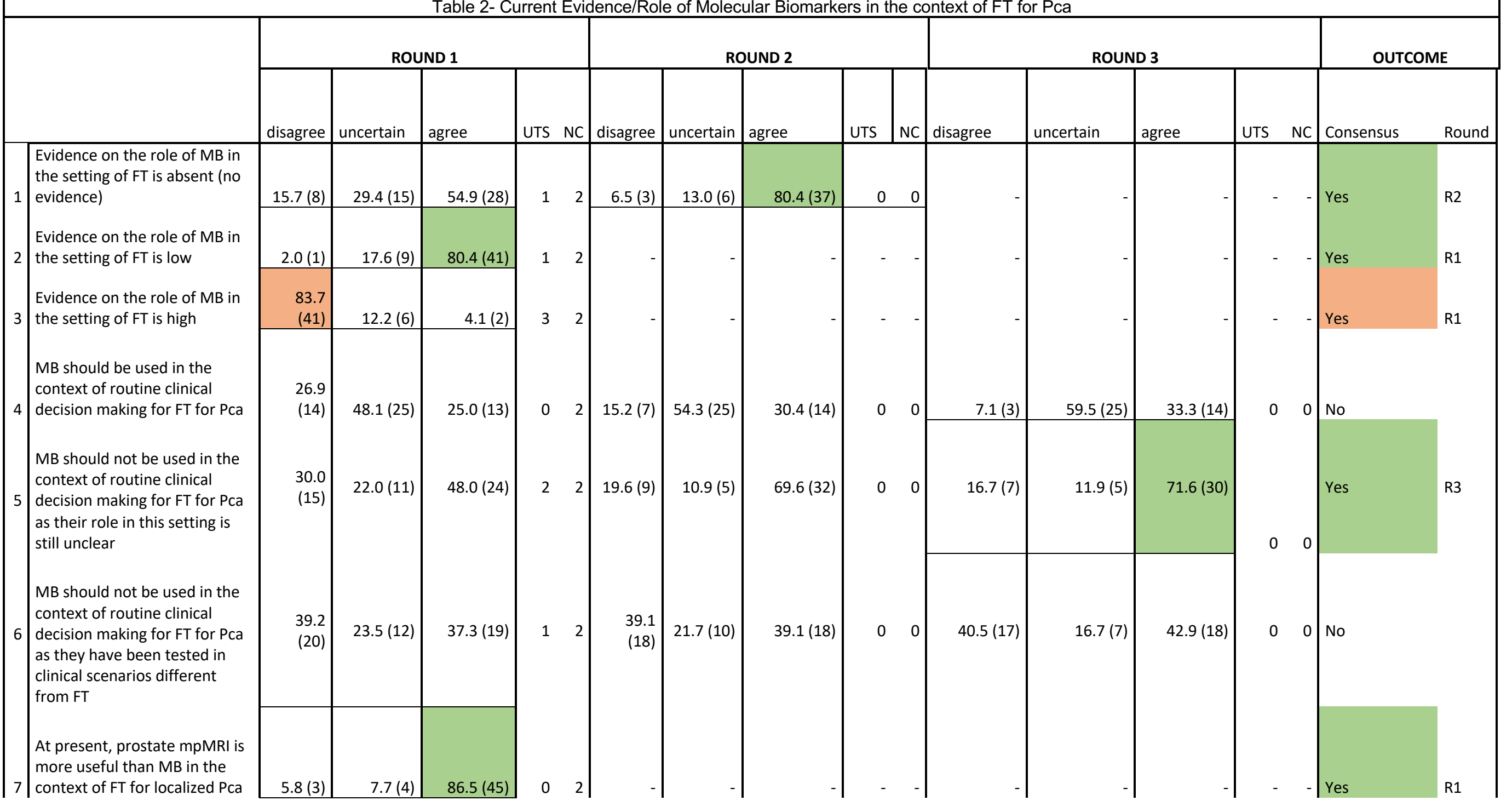




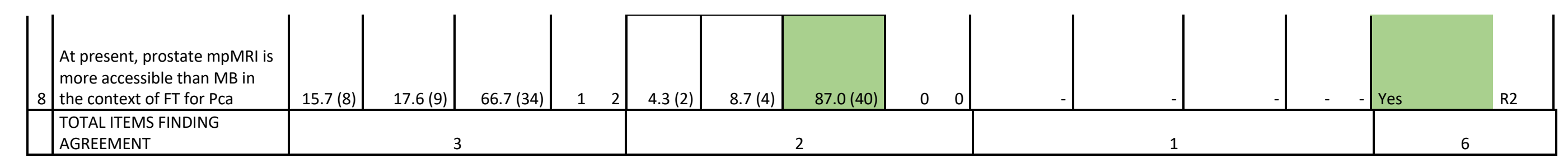




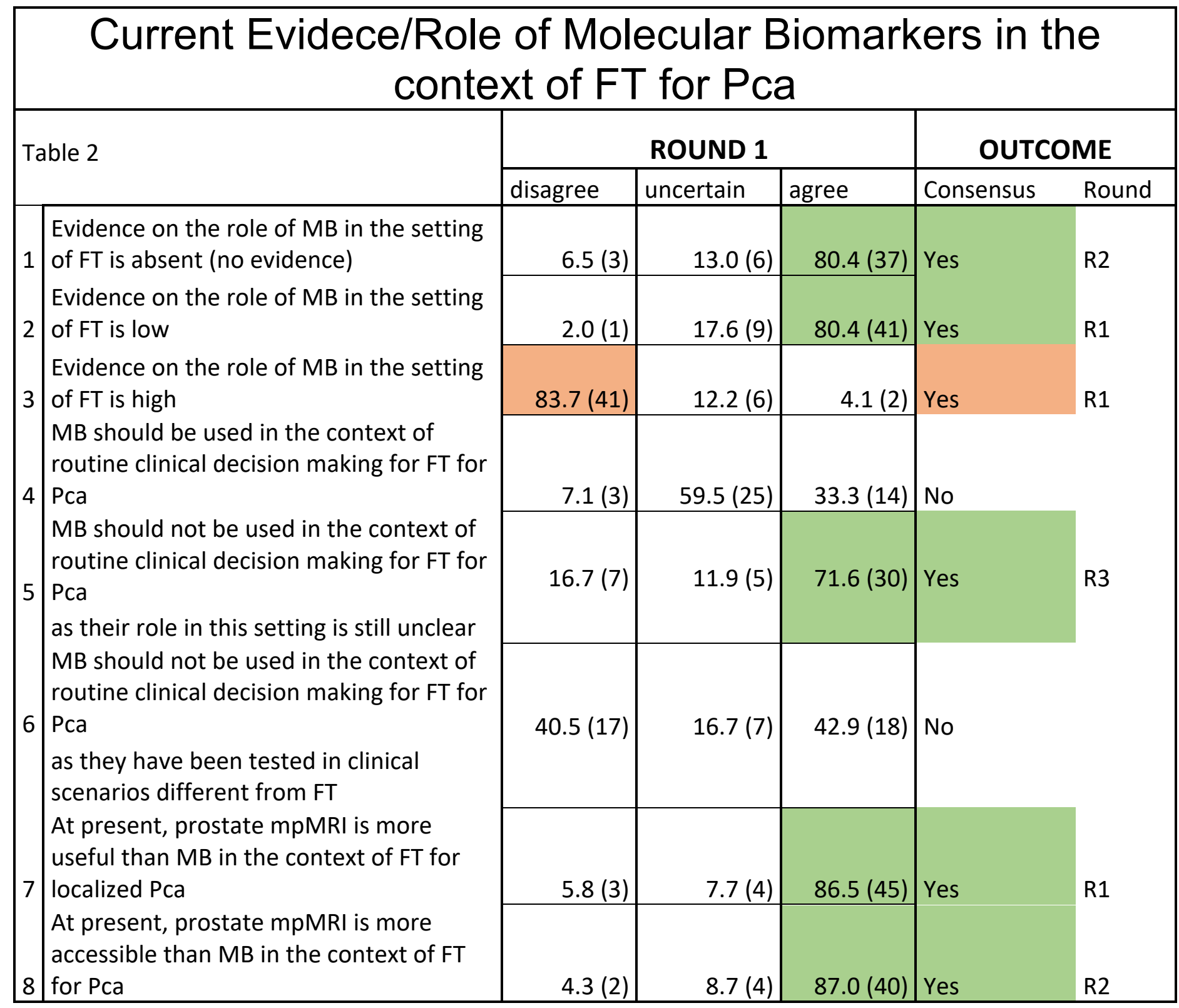


$A M B$ in the context of FT for Pca should be able to:

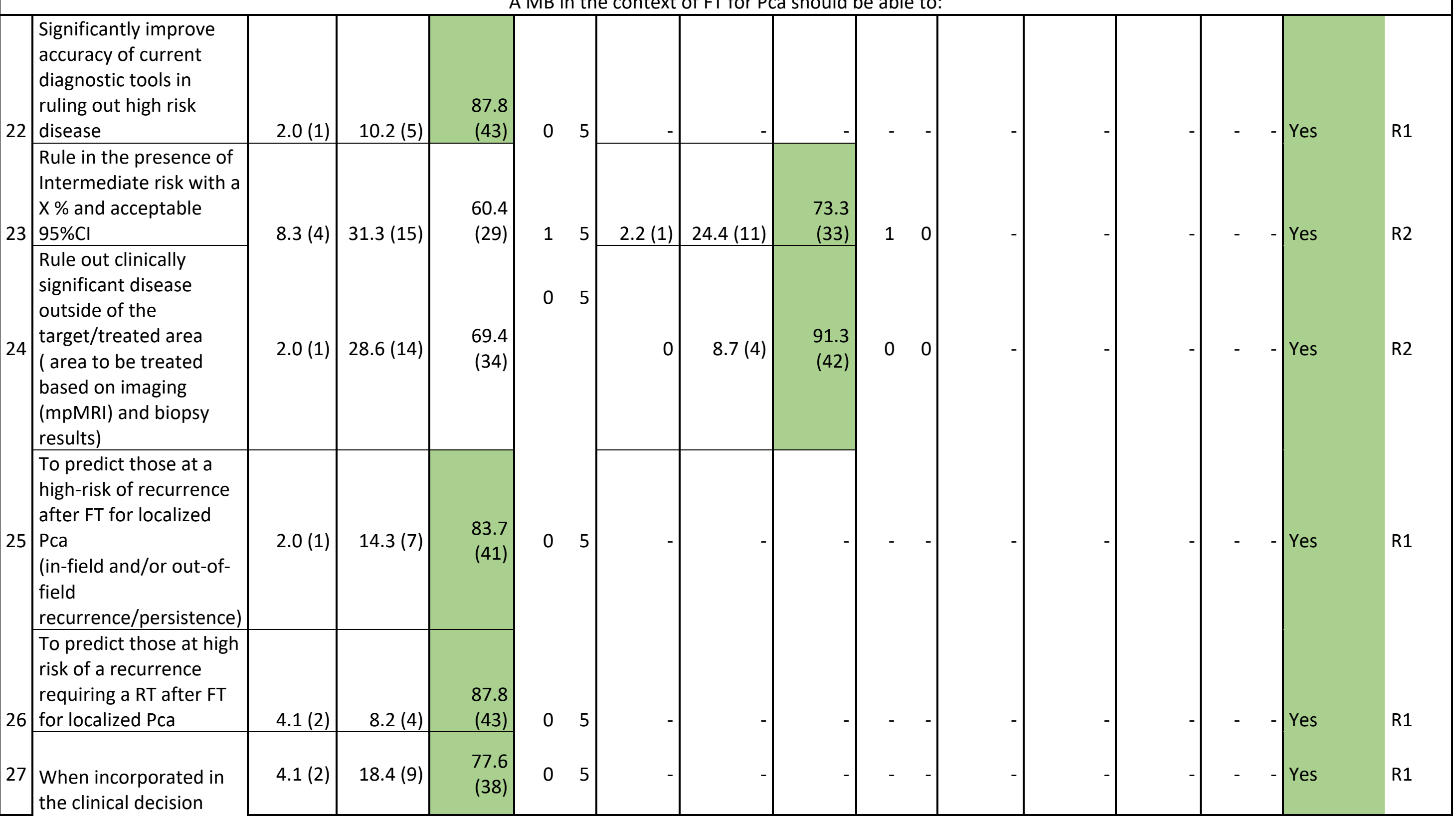




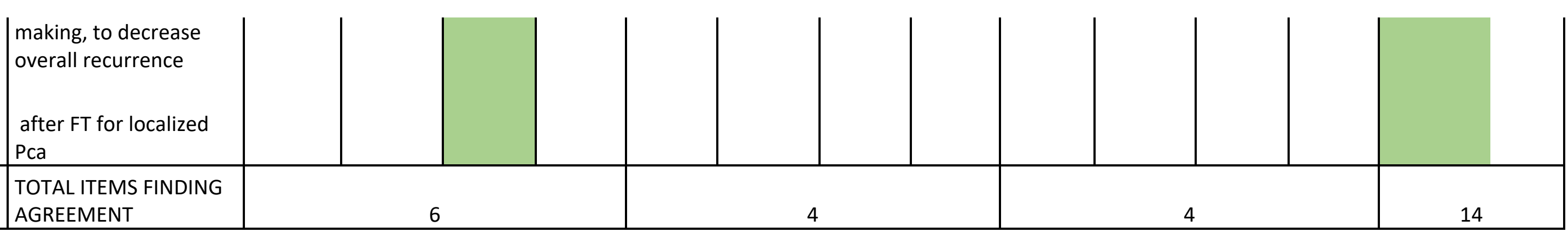




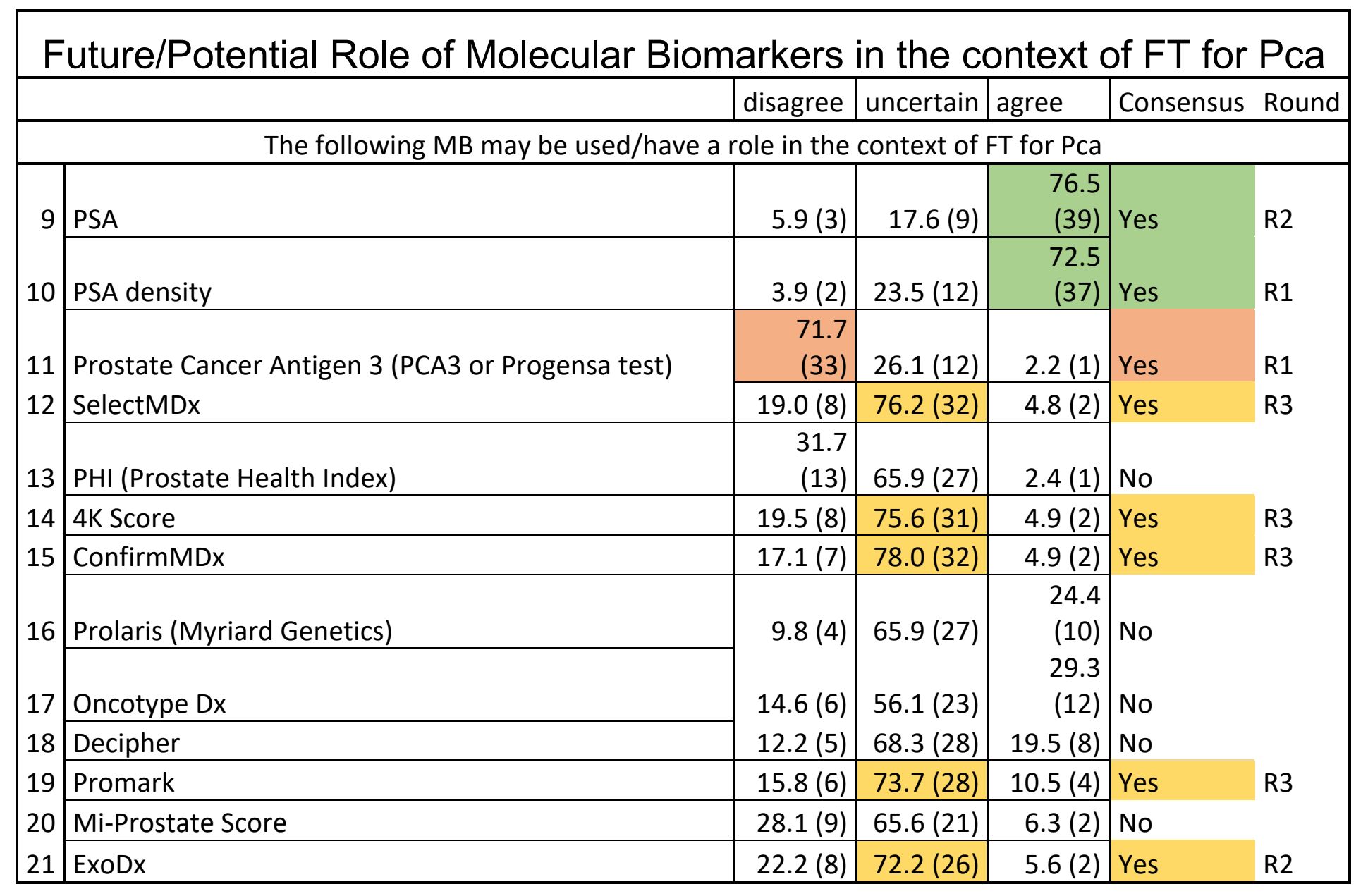




\section{Future/Potential Role of Molecular Biomarkers in the context of FT for Pca}

\begin{tabular}{|l|l|l|l|}
\hline disagree & uncertain & agree & Consensus Round \\
\hline
\end{tabular}

A MB in the context of FT for Pca should be able to:

\begin{tabular}{|c|c|c|c|c|c|c|}
\hline 22 & $\begin{array}{l}\text { Significantly improve accuracy of current diagnostic tools in ruling out high risk } \\
\text { disease }\end{array}$ & $2.0(1)$ & $10.2(5)$ & $\begin{array}{l}87.8 \\
(43)\end{array}$ & Yes & R1 \\
\hline 23 & Rule in the presence of Intermediate risk with a X \% and acceptable $95 \% \mathrm{Cl}$ & $2.2(1)$ & $24.4(11)$ & $\begin{array}{r}73.3 \\
(33) \\
\end{array}$ & Yes & $\mathrm{R} 2$ \\
\hline 24 & $\begin{array}{l}\text { Rule out clinically significant disease outside of the target/treated area } \\
\text { ( area to be treated based on imaging (mpMRI) and biopsy results) }\end{array}$ & 0 & $8.7(4)$ & $\begin{array}{l}91.3 \\
(42) \\
\end{array}$ & Yes & $\mathrm{R} 2$ \\
\hline 25 & $\begin{array}{l}\text { To predict those at a high-risk of recurrence after FT for localized Pca } \\
\text { (in-field and/or out-of-field recurrence/persistence) }\end{array}$ & $2.0(1)$ & $14.3(7)$ & $\begin{array}{l}83.7 \\
(41)\end{array}$ & Yes & R1 \\
\hline 26 & To predict those at high risk of a recurrence requiring a RT after FT for localized Pca & $4.1(2)$ & $8.2(4)$ & $\begin{array}{l}87.8 \\
(43) \\
\end{array}$ & Yes & R1 \\
\hline 27 & $\begin{array}{l}\text { When incorporated in the clinical decision making, to decrease overall recurrence } \\
\text { after FT for localized Pca }\end{array}$ & $4.1(2)$ & 18.4 (9) & $\begin{array}{l}77.6 \\
(38)\end{array}$ & Yes & R1 \\
\hline
\end{tabular}




\begin{tabular}{|c|c|c|c|c|c|c|c|c|c|c|c|c|c|c|c|c|c|c|}
\hline \multicolumn{19}{|c|}{$\begin{array}{c}\text { Important Tests/Exams to be included in future studies assessing the Role of Molecular } \\
\text { Biomarkers* }\end{array}$} \\
\hline \multirow{2}{*}{\multicolumn{2}{|c|}{ Table 4.}} & \multicolumn{5}{|c|}{ ROUND 1} & \multicolumn{5}{|c|}{ ROUND 2} & \multicolumn{5}{|c|}{ ROUND 3} & \multicolumn{2}{|c|}{ OUTCOME } \\
\hline & & disagree & uncertain & agree & UTS & $\mathrm{NC}$ & disagree & uncertain & agree & UTS $N$ & $\mathrm{NC}$ & disagree & uncertain & agree & UTS & $\mathrm{NC}$ & Consensus & Round \\
\hline 28 & PSA & $6.1(3)$ & $12.2(6)$ & $81.6(40)$ & 0 & 5 & & - & 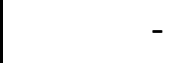 & - & - & - & - & - & - & - & Yes & R1 \\
\hline 29 & PSAd & $4.2(2)$ & $10.4(5)$ & $85.4(41)$ & 1 & 5 & - & - & 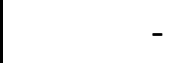 & - & - & - & - & - & - & - & Yes & $\mathrm{R} 1$ \\
\hline 30 & DRE & $36.7(18)$ & $32.7(16)$ & $30.6(15)$ & 0 & 5 & $43.5(20)$ & $23.9(11)$ & $32.6(15)$ & 0 & 0 & $47.6(20)$ & $19.0(8)$ & $33.3(14)$ & 0 & 0 & No & \\
\hline 31 & $\begin{array}{l}\text { Target biopsy (if } \\
\text { positive mpMRI) }\end{array}$ & 0 & $6.1(3)$ & $93.9(46)$ & 0 & 5 & - & - & - & - & & & & & & & Yes & R1 \\
\hline 32 & $\begin{array}{l}\text { Targeted and } \\
\text { systematic biopsy }\end{array}$ & 0 & $6.1(3)$ & $93.9(46)$ & 0 & 5 & & & . & - & 1 & - & -1 & & - & & Yes & $\mathrm{R} 1$ \\
\hline 33 & prostate mpMRI & 0 & 0 & $100.0(49)$ & 0 & 5 & - & - & - & - & & - & - & - & - & - & Yes & R1 \\
\hline 34 & $\begin{array}{l}\text { Prostate cancer } \\
\text { risk calculators }\end{array}$ & $6.1(3)$ & $46.9(23)$ & $46.9(23)$ & 0 & 5 & $4.3(2)$ & $30.4(14)$ & $65.2(30)$ & 0 & 0 & 0 & $11.9(5)$ & 88.1 (37) & 0 & 0 & Yes & R3 \\
\hline 35 & PSMA-PET & $26.5(13)$ & $30.6(15)$ & $42.9(21)$ & 0 & 5 & $21.7(10)$ & $21.7(10)$ & $56.5(26)$ & 0 & 0 & $11.9(5)$ & $19.0(8)$ & 69.0 (29) & 0 & 0 & No & \\
\hline 36 & Coline-PET & $53.1(26)$ & $36.7(18)$ & $10.2(5)$ & 0 & 5 & 73.9 (34) & $17.4(8)$ & $8.7(4)$ & 0 & 0 & - & - & - & - & - & Yes & $\mathrm{R} 2$ \\
\hline 37 & CT-scan & $67.3(33)$ & $26.5(13)$ & $6.1(3)$ & 0 & 5 & $87.0(40)$ & $8.7(4)$ & $4.3(2)$ & 0 & 0 & - & - & - & - & - & Yes & $\mathrm{R} 2$ \\
\hline 38 & Bone scan & $61.2(30)$ & $30.6(15)$ & $8.2(4)$ & 0 & 5 & $82.6(38)$ & $13.0(6)$ & $4.3(2)$ & 0 & 0 & - & - & - & - & - & Yes & $\mathrm{R} 2$ \\
\hline & $\begin{array}{l}\text { TOTAL ITEMS } \\
\text { FINDING } \\
\text { AGREEMENT }\end{array}$ & & 5 & & & & & 3 & & & & & 1 & & & & 9 & \\
\hline
\end{tabular}




\begin{tabular}{|c|c|c|c|c|c|c|}
\hline \multicolumn{7}{|c|}{$\begin{array}{l}\text { Tests/Exams to be included in studies assessing the Role of } \\
\qquad \mathrm{MB}^{*}\end{array}$} \\
\hline \multicolumn{2}{|c|}{ Table 4.} & \multirow{3}{*}{\begin{tabular}{|l} 
disagree \\
$6.1(3)$ \\
\end{tabular}} & \multirow{3}{*}{$\begin{aligned} \text { uncertain } \\
12.2(6)\end{aligned}$} & \multirow{3}{*}{\begin{tabular}{|c|}
$\mid$ agree \\
$81.6(40)$
\end{tabular}} & \multicolumn{2}{|c|}{ OUTCOME } \\
\hline & & & & & Consensus & Round \\
\hline 28 & PSA & & & & Yes & R1 \\
\hline 29 & PSAd & $4.2(2)$ & $10.4(5)$ & $85.4(41)$ & Yes & R1 \\
\hline 30 & DRE & $47.6(20)$ & $19.0(8)$ & $33.3(14)$ & No & \\
\hline 31 & Target biopsy (if positive mpMRI) & 0 & $6.1(3)$ & $93.9(46)$ & Yes & R1 \\
\hline 32 & Targeted and systematic biopsy & 0 & $6.1(3)$ & $93.9(46)$ & Yes & R1 \\
\hline 33 & prostate mpMRI & 0 & 0 & $100.0(49)$ & Yes & R1 \\
\hline 34 & Prostate cancer risk calculators & 0 & $11.9(5)$ & $88.1(37)$ & Yes & R3 \\
\hline 35 & PSMA-PET & $26.5(13)$ & $30.6(15)$ & $42.9(21)$ & No & \\
\hline 36 & Coline-PET & $73.9(34)$ & $17.4(8)$ & $8.7(4)$ & Yes & R2 \\
\hline 37 & CT-scan & $87.0(40)$ & $8.7(4)$ & $4.3(2)$ & Yes & R2 \\
\hline 38 & Bone scan & $82.6(38)$ & $13.0(6)$ & $4.3(2)$ & Yes & R2 \\
\hline
\end{tabular}


Table 5. Focal Therapy Society Statements on Molecular Biomarkers in the Context of Focal Therapy for Prostate Cancer.

- 1. Current Evidence/Role of MBs in the context of FT for localised PCa

1.1 Evidence on the role of MBs in the context of FT for PCa is absent (i.e. no evidence).

1.2 Currently/At Present, MBs should not be routinely [e.g. outside of research purposes] used in the context of FT for PCa as:

1.2.1 Their role in this setting is still unclear.

1.2.2 They have been tested in clinical scenarios different from FT.

1.3 At present, prostate mpMRI in the context of FT for PCa:

1.3.1 Is more useful than MBs.

1.3.2 Is more accessible than MBs.

- 2. Future/Potential Role of MBs in the context of FT for localised PCa:

2.1 MBs in the context of FT for PCa are a research priority*

2.2 MBs currently having a role in the context of FT for PCa:

2. 2.1 PSA.

2. 2.2 PSAD.

2.3 MBs not potentially having a role/not worth investigating in the context of FT for localised PCa:

2.3.1 PCA3 (Prostate Cancer Antigen 3 or Progensa Test).

2.4 MBs source:

2. 4.1 In a diagnostic setting (treatment selection) - there is not a preferred MBs source (urine, blood, tissue, others)*

2.4.2 In a follow-up setting (after FT) - non-tissue based MBs (urine, blood, others) may ideally be preferred*

2.5 Ideal features for MBs in the context of FT for localised PCa. MBs in the context of FT for localised PCa should be able to:

2.5.1 Significantly improve the accuracy of current diagnostic tools in ruling out a high risk disease.

2.5.2 Rule in the presence of an intermediate risk disease.

2.5.3 Rule out a clinically significant disease outside of the target/treated area (area to be treated based on imaging (mpMRI) and biopsy results).

2.5.4 Predict those at a high-risk of recurrence after FT (in-field and/or out-of-field recurrence/persistence).

2.5.5 Predict those at a high-risk of a recurrence/persistence requiring a radical treatment after FT.

2.5.6 When incorporated in clinical decision-making, to decrease an overall recurrence after FT.

- 3. Tests/Exams of Future Studies assessing the role of MBs in the context of FT for localised PCa 3.1 Future studies assessing the role of MBs in the context of FT for localised PCa should include the following tests/exams:

3.1.1 PSA.

3.1.2 PSAD.

3.1.3 mpMRI.

3.1.4 Target and Systematic Prostate Biopsies.

3.1.5 Prostate Cancer Risk Calculators.

3.1.6 DRE.

3.2 Future studies assessing the role of MBs in the context of FT for localised PCa should not include the following tests/exams:

3.2.1 Choline C-11 PET scan.

3.2.3 Bone scan.

3.2.2 CT scan.

3.3 There is interest for PSMA-PET in the context of FT for localised PCa and MBs. 